DEUTSCHE VIERTELJAHRS SCHRIFT FÜR

LITERATURWISSENSCHAFT UND

GEISTESGESCHICHTE 


\section{Die Phantasie des Neuen als Abduktion}

Von UwE WIRTH (Frankfurt a.M.)

\section{ABSTRACT}

Der Aufsatz beantwortet die Frage, ob es eine den Künsten und Wissenschaften gemeinsame Phantasie des Neuen gibt, im Rückgriff auf das Peircesche Konzept der Abduktion. Die Abduktion wird dabei zum einen als epistemologische „Strategie der Innovation “, zum anderen als „ästhetische Operation “ ausgezeichnet, die im Spannungsfeld von Assoziation, Einbildungskraft, Urteilskraft und Witz steht.

The essay aims at answering the question whether arts and sciences are backed by an unique Phantasy of Invention by referring to the peircean concept of abductive inference. Abductive inference is going to be highlightend as an epistemologic "strategy of innovation" as well as an "esthetic operation" defined by the interaction of association, imagination, power of judgement and wit.

Glaubt man Freud, so ist die Phantasie ein Surrogat des Spiels. Der Dichter tut "dasselbe wie das spielende Kind; er erschafft eine Phantasiewelt “ ${ }^{1}$ Dabei vollzieht sich das Phantasieren, mit Boris Groys zu sprechen, als „innovativer Tausch “, 2 der eine „Umwertung der Werte ${ }^{\text {“3 }}$ vornimmt und bestimmten „Strategien der Innovation “ folgt. ${ }^{4}$ Diese Strategien der Innovation möchte ich mit Blick auf die Frage, ob es eine den Künsten und Wissenschaften gemeinsame Phantasie des Neuen gibt, als Strategie der Abduktion fassen. In seinen Lectures on Pragmatism behauptet der Wissenschaftstheoretiker und Begründer der pragmatischen Semiotik, Charles Sanders Peirce, im Gegensatz zu Deduktion und Induktion sei die Abduktion die „einzige logische Operation, die irgendeine neue Idee einführt ". ${ }^{5}$ Im folgenden möchte ich die Abduktion als eine den Künsten und Wissenschaften gemeinsame Phantasie des Neuen beschreiben, die gleichermaßen als logische Operation, als phantastisches Gedankenspiel und als Gedankenblitz dargestellt werden kann. Zunächst wird es darum gehen, den Begriff des Neuen im Kontext der Epistemologie und unter Berücksichtigung abduktiven Folgerns zu bestimmen. In einem zweiten Schritt sollen die Anschlußmöglichkeiten der Abduktion an die ästhetischen und poetologischen Konzepte der Urteilskraft und des Witzes erörtert werden.

\footnotetext{
Sigmund Freud, Der Dichter und das Phantasieren, in: Studienausgabe, Frankfurt a.M. 1969 (1908), X, 172 .

${ }^{2}$ Boris Groys, Über das Neue, Frankfurt a.M. 1999 (1992), 146.

${ }^{3}$ Groys (Anm. 2), $66 \mathrm{f}$

${ }^{4}$ Groys (Anm. 2), 73.

5 Charles Sanders Peirce, Collected Papers, hrsg. Ch. Hartshorne, P. Weiß, Band I-VI (1931-1935), Band VII und VIII (1958), hrsg. A. W. Burks, Harvard University Press. Zitiert wird nach Band und Abschnitt. Hier: 5.171.
} 
Das Neue hat in der Geschichte der Erkenntnis- und Wissenschaftstheorie seine eigenen Schlüsselworte. Begriffe wie „Entdeckung“, „Erfindung“, „Einfall “ prägen den auf Invention und Innovation zielenden Duktus einer Epistemologie, der es um die Frage geht, auf welchem Wege wir zu neuem Wissen über die Welt gelangen können. Dabei steht die seit Descartes, Hume und Kant systematisch vorangetriebene Reflexion auf die Methoden und Prozeduren des Wissenserwerbs immer wieder vor dem Problem, dem Neuen in Form des „revolutionären Erkenntnissprungs“ oder der „kühnen Antizipation“ zwar eine zentrale epistemologische Rolle zuzuweisen, aber die Untersuchung der beim Entstehen neuer Erkenntnis involvierten Prozesse dezidiert auszuklammern.

Es ist ein epistemologischer Gemeinplatz, daß das Neue, also die „Entdekkung“, die „Erfindung “, der „Einfall“, nicht planbar ist. Schon in Lichtenbergs Aphorismen heißt es: „Alle Erfindungen gehören dem Zufall an, die eine näher, die andere weiter vom Ende, sonst könnten sich vernünftige Leute hinsetzen und Erfindungen machen, so wie man Briefe schreibt “ ${ }^{6}$ Die gleiche Auffassung vertritt die Wissenschaftstheorie des 20. Jahrhunderts. So schreibt Popper in seiner Logik der Forschung, daß es ,eine logische, rational nachkonstruierbare Methode, etwas Neues zu entdecken, nicht gibt “. ${ }^{7}$ Deshalb fordert er, daß wir „scharf zwischen dem Zustandekommen des Einfalls und den Methoden und Ergebnissen seiner logischen Diskussion unterscheiden " ${ }^{8}$ Auch Einstein ist wie Popper der Meinung, daß es zum Entdecken des Neuen keinen logischen W/eg gebe, „sondern nur die auf Einfühlung in die Erfahrung sich stützende Intuition “. 9 Da dieses „irrationale Moment" der „schöpferischen Intuition “ methodisch nicht planbar ist, kann es nicht Gegenstand der „Logik der Forschung“ sein. Dabei bezieht sich Popper auf Bergsons Intuitionsbegriff, der in seinen Vorlesungen La Pensée et le Mouvant zwei Arten von „intuitiver Klarheit“ („clarté intuitive“) des „unmittelbaren Bewußtseins“ unterscheidet. Die erste Form der Klarheit gründet nach Bergson darin, daß unser Verstand im Neuen nur Altgewohntes findet und sich daher in bekannten Gefilden bewegt: „Une idée neuve peut être claire parce qu'elle nous présente, simplement arrangées dans un nouvel ordre, des idées élementaires que nous possédions déjà “,${ }^{10}$ Die zweite Form von Klarheit dagegen ist die einer Idee „radicalement neuve et absolument simple, qui capte plus ou moins une intuition “. ${ }^{11}$ Durch radikal neue Ideen werden Probleme, die wir für unlösbar erachten, gelöst, um entweder zu

\footnotetext{
${ }^{6}$ Georg Christoph Lichtenberg, Aphorismen, Briefe, Schriften, Stuttgart 1953, 167.

7 Karl R. Popper, Logik der Forschung, Tübingen 1994 (1934), 7.

${ }^{8}$ Popper (Anm. 7), 6.

${ }^{9}$ Zit. nach Popper (Anm. 7), 7.

${ }^{10}$ Henri Bergson, La Pensée et le Mouvant. Essais et Conférences, Paris 1955, 31.

11 Bergson (Anm. 10), 31.
}

verschwinden oder um sich uns in anderer Weise zu stellen - „elles ont alors le double pouvoir d'éclairer le reste et de s'éclairer elles-mêmes “. ${ }^{12}$ Beide Formen „intuitiver Klarheit" werfen die selbe Frage auf, nämlich wie wir zu neuen Ideen kommen und wie wir neue Erkenntnis gewinnen können.

„Neue Erkenntnis“ kann dabei entweder bedeuten, etwas Neues zu beobachten, also mit einem „überraschenden Phänomen “ konfrontiert zu werden, oder aber für eine Reihe bekannter Tatsachen und Phänomene eine neue Theorie aufzustellen. Peirce schreibt mit Blick auf die Unterscheidung von „Entdekken “ und „Erfinden", ein Erkenntniszuwachs ("increase of information “) werde Entdeckung („Discovery“) genannt, auch wenn der alte Ausdruck „Invention" dafür sehr viel angemessener sei. Dadurch könne man nämlich den $\mathrm{Be}$ griff „Discovery“ für das „finding of a new thing - as the discovery of America" reservieren, während sich das Herausfinden einer neuen Eigenschaft an bereits bekannten Dingen als „Detection “ bezeichnen ließe. ${ }^{13}$ Die „Invention“ pendelt für Peirce zwischen den Polen des „Findens neuer Dinge“ und dem "Herausfinden neuer Eigenschaften“, wobei es eine beiden Aspekten gemein same „Logic of Discovery“ bzw. „Logic of Detection“ gibt: die Abduktion. Die Abduktion ist der "process of forming an explanatory hypothesis“. ${ }^{14}$ Ein Prozeß, im Rahmen dessen eine provisorische Synthetisierung von Prädikaten vorgenommen wird. Dabei bringt die abduktiv aufgestellte Hypothese „Mannigfaltiges zur Einheit "15 und bildet so die Grundlage plausibler und erklärungskräftiger Theorien: „the need of synthesizing a multitude of predicates [...] is the need of theory ". ${ }^{16} \mathrm{Da}$ die Abduktion die einzige Art von Argument ist, "which starts a new idea ", ${ }^{17}$ ist sie die einzige ,echt synthetische" Schlußform.

II.

Das philosophische Problem des Peirceschen Konzepts der Abduktion, verstanden als "Logic of Discovery“, besteht darin, daß sie erkenntniserweiternde, also neue, aber potentiell fallible Resultate hervorbringt, die logisch nicht zu rechtfertigen sind: Die einzige Rechtfertigung der Abduktion besteht darin, $\mathrm{da} ß$ „from its suggestion deduction can draw a prediction which can be tested

\footnotetext{
12 Bergson (Anm. 10), 32.

13 Peirce (Anm. 5), 2.430. Vgl. hierzu Kant, der in seiner Anthropologie in pragmatischer Hinsicht schreibt, eine Sache, die man entdeckt, werde „als vorher schon existierend angenommen, nur daß sie noch nicht bekannt war, z. B. Amerika vor Kolumbus“. (Immanuel Kant, Anthropologie in pragmatischer Hinsicht, in: Werkausgabe, hrsg. W. Weischedel, Frankfurt a.M. 1977, XII, 543).

${ }_{14}$ Peirce (Anm. 5), 5.171.

15 Peirce (Anm. 5), 5.276.

16 Peirce (Anm. 5), 3.516.

17 Peirce (Anm. 5), 2.96.
} 
by induction" und daß, ,if we are ever to learn anything or to understand phenomena at all, it must be by abduction ". ${ }^{18}$ Ausgerechnet der für alles Verstehen grundlegende Schlußmodus der Abduktion entzieht sich somit der Kontrolle der deduktiven Logik. ${ }^{19}$ Die Abduktion ist lediglich eine Antizipation künftiger Begründbarkeit.

Anders als in Poppers Logik der Forschung wird beim Peirceschen Abduktionskonzept keine scharfe Grenze zwischen der psychologischen und der logischen Domäne des „Context of Discovery“ gezogen. Die Erkenntnistheorie à la Popper untersucht als Erkenntnislogik lediglich die Methoden der Überprüfung, der jeder Einfall, der ernst genommen werden soll, zu unterwerfen ist. Die Vorgänge des Aufstellens von Hypothesen, also die Prozesse, wie Neues entsteht, können dagegen nur empirisch-psychologisch untersucht werden und haben "mit Logik wenig zu tun ". ${ }^{20}$ Ganz anders argumentiert Peirce: Er behauptet, daß die Abduktion, obwohl sie nur sehr wenig durch logische Regeln behindert werde, eine Form logischer Inferenz sei, „asserting its conclusion only problematically or conjecturally, it is true, but nevertheless having a perfectly definite logical form". ${ }^{21}$ Diese scheinbar paradoxe Doppelbestimmung der Peirceschen „Logic of Discovery“ als nur wenig von logischen Regeln behin derter, innovativer Schluß, der dennoch „eine vollkommen bestimmte logische Form besitzt", ist der Auslöser einer bis heute andauernden philosophischen Diskussion. ${ }^{22}$ Dabei muß zwischen zwei Fragen unterschieden werden. Zum einen die Frage, inwiefern die Abduktion tatsächlich eine gültige Form logischen

18 Peirce (Anm. 5), 5.171.

19 Peirce (Anm. 5), 6.485.

20 Vgl. Popper (Anm.7), 7

${ }^{21}$ Peirce (Anm. 5), 5.188.

${ }^{22}$ Vgl. hierzu Hansons Aufsatz „The Logic of Discovery“, wo dieser im Rekurs auf Popper und Reichenbach die Analogie zwischen "hypothetisch-deduktiver Methode" und „retroduktiver Inferenz" bildet. Wie Popper kommt auch Hanson zu dem Schluß, daß es letztlich keine Möglichkeit einer philosophisch begründeten „Entdeckungslogik“ gibt (vgl. Norwood Russel Hanson, „The Logic of Discovery“, The Journal of Philosophy 55 [1958], 1073-1089, hier: 1074ff.). Zugleich zeigt Hanson jedoch, daß sich der Peircesche Ansatz der Abduktion zur Formulierung forschungsstrategischer "Leitprinzipien " eignet. Die Abduktion wird zu einer Strategie, auf "die beste Erklärung " zu schlieBen. Auch Fann folgt in Peirce's Theory of Abduction Poppers Ausgrenzung des abduktiven Einfalls aus der Forschungslogik und wendet sich statt dessen der Frage zu, in welcher Form abduktives Folgern die Hypothesenbildung („hypothesis formation “) und die Hypothesenauswahl („hypothesis selection “) betreibt (vgl. Kuang T. Fann, Peirce's Theory of Abduction, The Hague 1970, 7). Neuere Untersuchungen zum Thema finden sich in Tomis Kapitan, „In What Way is Abductive Inference Creative? “, Transactions of the Charles Sanders Peirce Society 26 (1990), 499-512 sowie in Ansgar Richter, Der Begriff der Abduktion bei Charles Sanders Peirce, Frankfurt a.M., Berlin, Bern, New York, Paris, Wien 1995
Schließens ist, zum anderen die Frage, inwieweit die Abduktion eine innovative Strategie des Denkens darstellt.

Das Konzept der Abduktion ist die Antwort des Peirceschen Pragmatismus auf die kantische Frage nach der Möglichkeit „synthetischer Urteile a priori“ An die Stelle des kantischen „transzendentalen Bewußtseins“ als „höchster Punkt" des Erkenntnisvermögens tritt, wie Karl-Otto Apel in Transformation der Philosophie schreibt, der "finale Interpretant" der indefiniten „Community of Investigators ". ${ }^{23}$ Diese Transformation der transzendentalen Logik in eine „Logic of Science“ führt dazu, „daß der Verstand gewissermaßen der Natur das Gesetz - in the long run - vorschreiben kann, ohne sie daran zu hindern, ihrerseits den Inhalt aller nur denkbaren synthetischen Sätze auf dem Wege des äußeren Erfahrungszwanges bestimmen zu können ". ${ }^{24}$ Peirce transformier mit Hilfe der synthetischen Schlußweise der Abduktion das transzendentale a priori in ein pragmatisches a posteriori, und so kommt er zu dem Schluß: „If you carefully consider the question of pragmatism you will see that it is nothing else than the question of the logic of abduction “ ${ }^{25}$ Dabei erlaubt der Pragmatismus, „jeden Flug der Imagination, vorausgesetzt, daß diese Imagination schließlich eine mögliche praktische Wirkung erhellt" ${ }^{26}$ Die Abduktion dient dazu, sich plausible Theorien vorzustellen und neue Theorien zu erfinden. Die Abduktion nimmt eine „creation of hypothesis" vor, die als "grand work of poietic genius" erscheint, ${ }^{27}$ auch wenn sie nicht den Geltungsanspruch der Wissenschaftlichkeit reklamieren kann, da sich ihr noch kein Wahrheitswert zuschreiben läßt. Die Abduktion ist so besehen ein nützliches Vorspiel („a useful prelude“) der eigentlichen Wissenschaft. ${ }^{28}$

Das „Spiel der Wissenschaft" besteht darin, die „Fragen an die Natur" in Form hypothetischer Wett-Fragen zu formulieren, auf die das Experiment die Antwort geben soll. ${ }^{29}$ In diesem Spiel geht es sowohl um das Lösen vorhandener Probleme als auch um das Formulieren neuer Probleme. Da für Peirce das Ziel der Wissenschaft der Erfolg und nicht das korrekte Erfüllen methodischer Vorschriften ist, lautet seine erste Regel des Denkens: „Do not block the way of inquiry“. ${ }^{30}$ Die Peircesche Formel erinnert an Feyerabends "anything goes" zumal er die wissenschaftliche Methode als ${ }_{n}$ the general method of sucessful

${ }^{23}$ Vgl. Karl-Otto Apel, Transformation der Philosophie, Band1, Frankfurt a.M. $1973,173$.

${ }^{24}$ Karl-Otto Apel, Der Denkweg des Charles Sanders Peirce, Frankfurt a.M. 1967, 75 .

${ }^{25}$ Peirce (Anm. 5), 5.196.

26 Peirce (Anm. 5), 5.196.

27 Peirce (Anm. 5), 4.238.

28 Peirce (Anm. 5), 1.235 .

29 Peirce (Anm. 5), 1.120.

${ }^{30}$ Peirce (Anm. 5), 1.135 . 
scientific research“ definiert. ${ }^{31}$ Eben deshalb kann die Abduktion als „Strategie der Innovation “ gewertet werden, die die Phantasie des Neuen spielerisch pragmatisiert: Sie dient dem Entdecken eines relevanten, bislang noch nicht berücksichtigten Aspekts, der sich kohärent und einfach, mithin plausibel, in einen theoretischen Zusammenhang integrieren und ökonomisch überprüfen läßt.

Das spezifisch pragmatische Moment der "Logic of Discovery“ ist der Rekurs auf ein "Principle of Economy“. ${ }^{2}$ Die „leitende Überlegung“ des abduktiven Hypothesenaufstellens ist für Peirce - hier bezieht er sich auf Ernst Mach die Frage der Ökonomie, „der Ökonomie des Geldes, der Zeit, des Denkens und der Energie". ${ }^{33}$ Das Prinzip der Ökonomie betrifft die Relation „between utility and cost " ${ }^{34}$ es ist eine Strategie im Foucaultschen Sinne, genau genommen sogar ein Dispositiv, das der „Optimierung“ des Erwerbs von Wissen dient. ${ }^{35}$ Die Kosten betreffen den Aufwand, mit dem im Rahmen des Erkenntnisprozesses Fehler und Irrtum beseitigt werden können, um den Wert und den Nutzen des gewonnenen Wissens zu steigern. ${ }^{36} \mathrm{Um}$ im Bild der Wette zu bleiben: Der Forschungsaufwand ist der „Einsatz“, der zu einem Erkenntnisgewinn führen soll. Dieser variiert je nachdem, ob die Wette im Rahmen der „normalen Wissenschaft" die Lösung eines Rätsels prognostiziert oder aber als Erkenntnissprung eine revolutionäre Neuentdeckung macht, die die alten Rätsel durch neue ersetzt.

Nach Kuhn dienen die "Spielregeln" der "normalen Wissenschaft" dazu, den Punkt zu verzögern, an dem das Paradigma geändert wird, um „ein neues Rätsel zu definieren und das alte nicht zu lösen “. ${ }^{37}$ Während die ,normale Wissenschaft" eine „höchst determinierte Tätigkeit“ ist, ${ }^{38}$ geht vielen neuen „revolutionären" Theorien und Entdeckungen „nicht etwa ein Nichtwissen voraus, sondern die Erkenntnis, daß mit den vorhandenen Kenntnissen etwas nicht in Ordnung ist ". ${ }^{39} \mathrm{Zu}$ einem Paradigmenwechsel kommt es daher nur, wenn sich

31 Peirce (Anm. 5), 7.79.

32 Peirce (Anm. 5), 5.600

${ }_{33}$ Peirce (Anm. S), 5.600

${ }^{34}$ Peirce (Anm. S), 7.140.

35 Vgl. Michel Foucault, Uberwachen und Strafen. Die Geburt des Gefängnisses, Frankfurt a.M. 1994 (1975), 280.

${ }_{36}$ Dieses Prinzip der Ökonomie bestimmt den Diskurs der Wissenschaft - aber auch alle anderen Formen methodischen Interpretierens. So schreibt der Wissenschaftstheoretiker Nicholas Rescher, die „Evolution des Wissens“ folge der Dynamik „ökonomischer Rationalität", weshalb sich genau die Verfahren durchsetzten, „die kosteneffizient sind " (Nicholas Rescher, Warum sind wir nicht klüger? Der evolutionäre Nutzen von Dummheit und Klugheit, übersetzt von A. und H. Pape, Stuttgart 1994, 40).

37 Thomas S. Kuhn, Die Struktur wissenschaftlicher Revolutionen, Frankfurt a.M. 1976 (1962), 53.

38 Kuhn (Anm. 37), 56.

39 Thomas S. Kuhn, Die Entstehung des Neuen, Frankfurt a.M. 1978, 319. eine Theorie in Folge von Fachkrisen als unfähig erweist, „Regeln zur Bestimmung lösbarer Rätselaufgaben bereitzustellen“ “.40 Die Folge eines Paradigmenwechsels ist eine neue Perspektive, denn er veranlaßt die Wissenschaftler, „die Welt ihres Forschungsbereichs anders zu sehen [...]. Unter der Führung eines neuen Paradigmas verwenden die Wissenschaftler neue Apparate und sehen sich nach neuen Dingen um". 41

Signifikanterweise ist die Peircesche Erkenntnistheorie sowohl mit Poppers als auch mit Kuhns Auffassungen kompatibel. Einerseits sieht Peirce wie Popper die „Evolution des Wissens" als Selektionsprozeß, in dem sich durch „Conjecture and Refutation" die "fittere Hypothese" gleichsam herausmendelt. ${ }^{42}$ Zugleich problematisiert Peirce wie Kuhn den „kritischen Punkt“ jeder Erkenntnistheorie, nämlich den Punkt, an dem alte Probleme in neue Probleme transformiert werden. Im Rahmen der "normalen Wissenschaft" wird das Neue nur als „überraschendes Phänomen " wahrgenommen, das im Kontrast zum Erwartungshorizont der community of investigators steht: „Of course, nothing can appear as definitely new without being contrasted with a background of the old“. Deshalb gilt: „The first new feature of this first surprise is, for example, that it is a surprise “. ${ }^{43}$ Die Aufgabe abduktiven Schließens besteht nun darin, eine Theorie zu erfinden, die eine plausible Erklärung dafür liefert, warum das beobachtete Phänomen als überraschendes wahrgenommen wird. ${ }^{44}$ Daher sucht der Forscher nach einer Perspektive, von der aus sich das überraschende, rätselhafte Phänomen nicht mehr als rätselhaft erweist. ${ }^{45}$ In seinem Artikel Guessing schreibt Peirce, der Erinnerung an die beobachteten, rätselhaften Tatsachen folge das Bemühen, "to rearrange them, to view them in such new perspective, that the unexpected experience shall no longer appear surprising “. ${ }^{46}$ Diese veränderte Sichtweise gegenüber einer zunächst überraschenden Erfahrung gibt der neuen Theorie ihre explanative Kraft: „At length a conjecture arises that furnishes a possible Explanation ". ${ }^{47}$ Die Herangehensweise des Forschers besteht also darin, seine Beobachtung einerseits als Konsequenz einer unbekannten Ursache, andererseits als Teil eines größeren Zusammenhangs zu betrachten. Das Neuarrangement der Beobachtung wird vollzogen, indem man den überraschenden Phänomenen eine pars pro toto Rolle zu-

${ }^{40}$ Kuhn (Anm. 39), 371.

${ }^{41}$ Kuhn (Anm. 37), 123.

42 Karl R. Popper, Objective Knowledge. An Evolutionary Approach, Oxford 1979 (1972), 241. Vgl. aber auch ders., Conjectures and Refutations. The Growth of Scientific Knowledge, London 1969 (1963).

${ }^{43}$ Peirce (Anm. 5), 7.188.

${ }_{44}$ Peirce (Anm. 5), 2.776.

45 Vgl. Peirce (Anm. 5), 6.469.

46 Charles Sanders Peirce, Guessing, in: The Hound and Horn (1929), 267-285, hier: 267.

${ }^{47}$ Peirce (Anm. S), 6.469 
weist: „[T]he surprising facts that we have observed are only one part of a larger system of facts, of which the other part has not come within the field of our experience". ${ }^{48}$ Die Forschung zielt mithin darauf ab, den Zusammenhang mit einem „größeren System von Tatsachen" zu rekonstruieren, das noch außerhalb des Erfahrungshorizontes derjenigen liegt, die das überraschende Phänomen als überraschendes Phänomen wahrgenommen haben. Was Peirce hier formuliert ist nichts anderes als das hermeneutische Problem des „Neu- und Andersverstehens".

III.

Die Aufgabe der Abduktion als erster Schritt des Erkenntnisprozesses besteht zum einen im aneignenden Verstehen von Neuem, das zunächst als „überraschendes, erklärungsbedürftiges Phänomen " erscheint, zum anderen im paradigmatischen „Neu-Verstehen“, also im Erfinden einer neuen Theorie. Interessanterweise treten diese beiden Aspekte im Rahmen des Abduktionskonzepts nicht als konkurrierende, sondern als komplementäre Momente des Forschungs- und Interpretationsprozesses auf.

Das verdächtige oder plötzliche Einbrechen einer erklärungsbedürftigen Tatsache in den Erwartungs- und Erfahrungshorizont des Interpreten bestimmt die innere Form des abduktiven Folgerns, die Peirce folgendermaßen charakterisiert:

„Die überraschende Tatsache $\mathrm{C}$ wird beobachtet; aber wenn A wahr wäre, würde C eine Selbstverständlichkeit sein; folglich besteht Grund zu vermuten, daß A wahr ist". 49

Im Original heißt es: „hence there is reason to suspect that A is true “. Die Annahme, daß das Antezedens $A$ möglicherweise zur Conclusio $C$ führen könnte, beruht auf einem Verdacht seitens des Schlußfolgernden. Für Peirce ist der Verdacht (,suspicion“) ein „,irrationales Gefühl“ (,irrational feeling“), welches eine Reaktion auf die Überraschung darstellt, daß sich eine Erwartung als irrig herausgestellt hat. Der Verdacht entspricht dem Gefühl des Zweifels („doubt“) an einer Gewohnheit. Umgekehrt entspricht das plötzliche Gefühl des „Eureka“, die Lösung eines Problems gefunden zu haben, dem Wechsel zu einer neu en Gewohnheit. ${ }^{50}$ Die Abduktion schließt von einem überraschenden Phänomen auf dessen Ursache zurück, wobei sie zugleich die argumentative Begründbarkeit des angenommenen Ursache-Wirkungs-Verhältnisses miteinbezieht, um eine plausible Theorie zu formulieren. Dies geschieht in Form spielerischen Grübelns, in dessen Verlauf ein abduktives Argument zusammengepuzzelt

48 Peirce (Anm. 46), 267

49 Peirce (Anm. 5), 5.189

so Vgl. Peirce (Anm. 5), 8.270. wird. Die Form abduktiven Schließens ist die eines „umgedrehten“ bzw. „verkehrten" modus ponens. Beim quasi-deduktiven modus ponens wird die Regel "Wenn A, dann C“ vorausgesetzt und vom eingetretenen Fall „daß A “ auf die Konklusion „daher $C$ " geschlossen. Der modus ponens stellt sich mithin als Schluß von einer vorausgesetzten konditionalen Regel und einer Tatsache dar, die als Nachsatz in der Regel vorkommt. Die Abduktion dagegen ist vom logischen Standpunkt aus gesehen "reasoning from consequent to antecedent ", 51 also ein Rückschluß, eine „Retroduction“, von der Konklusion auf die Prämissen. Die überraschende Tatsache wird als Konsequenz $C$ eines noch unbekannten Antezedens $A$ betrachtet, wobei $A$ rückschlüssig ermittelt, d. h. entweder rekonstruiert oder neu-konstruiert werden muß. Diese abduktiv aufgestellte $\mathrm{Hy}$ pothese muß anschließend deduktiv auf ihre logische und induktiv auf ihre empirische Richtigkeit geprüft werden.

Angenommen, wir kämen in einen Raum, in dem ein gefüllter Sack liegt, daneben ein Haufen weißer Bohnen. Wir könnten auf die Idee kommen, eine assoziative Verbindung zwischen den Bohnen und dem gefüllten Sack herzustellen, ja, wir könnten sogar so weit gehen zu mutmaßen, daß alle Bohnen in diesem Sack weiß sind. Ihre logische Gültigkeit muß diese Hypothese beweisen, sobald sie im Rahmen einer Deduktion dargestellt wird. Ihre empirische Richtigkeit kann dagegen nur im Rahmen eines Experiments belegt werden, nämlich indem man in den Sack hineingreift, also induktiv tätig wird. Die Abduktion besteht in der Idee, eine assoziative Verbindung zwischen Bohnen und Sack herzustellen und diese Idee so zu formulieren, daß sie sich als Hypothese im Rahmen einer rationalen Argumentation überprüfen läßt. Das heißt, die Abduktion leistet die Transformation einer Assoziation in eine Argumentation, wobei sie die assoziierten Propositionen und Textteile wie Prämissen organisiert, also die Zwischenräume nicht nur als Kontiguitätsbeziehung interpretiert, sondern als inferentielle Verknüpfungen. Durch diesen „innovativen Tausch" 52 zwischen assoziativen und argumentativen Rollen wird eine „Umwertung der Werte ${ }^{\text {53 }}$ vollzogen. Die Assoziationen werden nicht nur neu arrangiert, sondern in einen argumentativen Rahmen integriert. Die Abduktion nimmt einen Rahmenwechsel, eine Modulation im Sinne Goffmans vor. ${ }^{54}$

Genau genommen handelt es sich sogar um einen doppelten Rahmenwechsel, da die Abduktion von einer erlebten zeitlichen Relation simultan auf eine logische Relation innerhalb der Denkwelt und auf eine hypothetische kausale Relation innerhalb der äußeren Erfahrungswelt schließt. Der abduktive Prozeß ist ein Vorgriff auf einen logischen Begründungszusammenhang, der sich im

\footnotetext{
51 Peirce (Anm. 5), 6.469.

$\$ 2$ Groys (Anm. 2), 146

53 Groys (Anm.2), $66 \mathrm{f}$

$54 \mathrm{Vgl}$. Erving Goffman, Rahmen-Analyse, Ein Versuch über die Organisation von Alltagserfahrungen, Frankfurt a.M. 1996 (1974), $55 \mathrm{f}$.
} 
weist: „[T] he surprising facts that we have observed are only one part of a larger system of facts, of which the other part has not come within the field of our experience ". ${ }^{48}$ Die Forschung zielt mithin darauf ab, den Zusammenhang mit einem „größeren System von Tatsachen“ zu rekonstruieren, das noch außerhalb des Erfahrungshorizontes derjenigen liegt, die das überraschende Phänomen als überraschendes Phänomen wahrgenommen haben. Was Peirce hier formuliert ist nichts anderes als das hermeneutische Problem des „Neu- und Andersverstehens".

III.

Die Aufgabe der Abduktion als erster Schritt des Erkenntnisprozesses besteht zum einen im aneignenden Verstehen von Neuem, das zunächst als „überraschendes, erklärungsbedürftiges Phänomen " erscheint, zum anderen im paradigmatischen „Neu-Verstehen “, also im Erfinden einer neuen Theorie. Interessanterweise treten diese beiden Aspekte im Rahmen des Abduktionskonzepts nicht als konkurrierende, sondern als komplementäre Momente des Forschungs- und Interpretationsprozesses auf.

Das verdächtige oder plötzliche Einbrechen einer erklärungsbedürftigen Tatsache in den Erwartungs- und Erfahrungshorizont des Interpreten bestimmt die innere Form des abduktiven Folgerns, die Peirce folgendermaßen charakterisiert:

„Die überraschende Tatsache $\mathrm{C}$ wird beobachtet; aber wenn A wahr wäre, würde C eine Selbstverständlichkeit sein; folglich besteht Grund zu vermuten, $\mathrm{daß}$ A wahr ist". 49

Im Original heißt es: „hence there is reason to suspect that A is true“. Die Annahme, daß das Antezedens A möglicherweise zur Conclusio $C$ führen könnte, beruht auf einem Verdacht seitens des Schlußfolgernden. Für Peirce ist der Verdacht ( „suspicion“) ein „,irrationales Gefühl“ (,irrational feeling“), welches eine Reaktion auf die Überraschung darstellt, daß sich eine Erwartung als irrig herausgestellt hat. Der Verdacht entspricht dem Gefühl des Zweifels ( doubt $^{\text {“ }}$ an einer Gewohnheit. Umgekehrt entspricht das plötzliche Gefühl des „Eureka“, die Lösung eines Problems gefunden zu haben, dem Wechsel zu einer neuen Gewohnheit. ${ }^{50}$ Die Abduktion schließt von einem überraschenden Phänomen auf dessen Ursache zurück, wobei sie zugleich die argumentative Begründ barkeit des angenommenen Ursache-Wirkungs-Verhältnisses miteinbezieht, um eine plausible Theorie zu formulieren. Dies geschieht in Form spielerischen Grübelns, in dessen Verlauf ein abduktives Argument zusammengepuzzelt

48 Peirce (Anm. 46), 267.

49 Peirce (Anm. 5), 5.189.

so Vgl. Peirce (Anm. 5), 8.270. wird. Die Form abduktiven Schließens ist die eines „umgedrehten“ bzw. „verkehrten" modus ponens. Beim quasi-deduktiven modus ponens wird die Regel "Wenn $A$, dann $C$ “ vorausgesetzt und vom eingetretenen Fall „da $\beta A$ " auf die

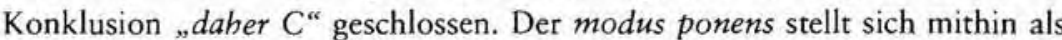
Schluß von einer vorausgesetzten konditionalen Regel und einer Tatsache dar, die als Nachsatz in der Regel vorkommt. Die Abduktion dagegen ist vom logischen Standpunkt aus gesehen "reasoning from consequent to antecedent ", 51 also ein Rückschluß, eine „Retroduction“, von der Konklusion auf die Prämissen. Die überraschende Tatsache wird als Konsequenz $C$ eines noch unbekannten Antezedens $A$ betrachtet, wobei $A$ rückschlüssig ermittelt, d.h. entweder rekonstruiert oder neu-konstruiert werden muß. Diese abduktiv aufgestellte $\mathrm{Hy}$ pothese muß anschließend deduktiv auf ihre logische und induktiv auf ihre empirische Richtigkeit geprüft werden.

Angenommen, wir kämen in einen Raum, in dem ein gefüllter Sack liegt, daneben ein Haufen weißer Bohnen. Wir könnten auf die Idee kommen, eine assoziative Verbindung zwischen den Bohnen und dem gefüllten Sack herzustellen, ja, wir könnten sogar so weit gehen zu mutmaßen, daß alle Bohnen in diesem Sack weiß sind. Ihre logische Gültigkeit muß diese Hypothese beweisen, sobald sie im Rahmen einer Deduktion dargestellt wird. Ihre empirische Richtigkeit kann dagegen nur im Rahmen eines Experiments belegt werden, nämlich indem man in den Sack hineingreift, also induktiv tätig wird. Die Abduktion besteht in der Idee, eine assoziative Verbindung zwischen Bohnen und Sack herzustellen und diese Idee so zu formulieren, daß sie sich als Hypothese im Rahmen einer rationalen Argumentation überprüfen läßt. Das heißt, die Abduktion leistet die Transformation einer Assoziation in eine Argumentation, wobei sie die assoziierten Propositionen und Textteile wie Prämissen organisiert, also die Z wischenräume nicht nur als Kontiguitätsbeziehung interpretiert, sondern als inferentielle Verknüpfungen. Durch diesen „innovativen Tausch" 52 zwischen assoziativen und argumentativen Rollen wird eine „Umwertung der Werte ${ }^{\text {“53 }}$ vollzogen. Die Assoziationen werden nicht nur neu arrangiert, sondern in einen argumentativen Rahmen integriert. Die Abduktion nimmt einen Rahmenwechsel, eine Modulation im Sinne Goffmans vor. ${ }^{54}$

Genau genommen handelt es sich sogar um einen doppelten Rahmenwechsel, da die Abduktion von einer erlebten zeitlichen Relation simultan auf eine logische Relation innerhalb der Denkwelt und auf eine hypothetische kausale Relation innerhalb der äußeren Erfahrungswelt schließt. Der abduktive Prozeß ist ein Vorgriff auf einen logischen Begründungszusammenhang, der sich im

\footnotetext{
${ }^{51}$ Peirce (Anm. 5), 6.469

52 Groys (Anm. 2), 146

53 Groys (Anm. 2), $66 \mathrm{f}$

$54 \mathrm{Vgl}$. Erving Goffman, Rahmen-Analyse. Ein Versuch über die Organisation von Alltagserfahrungen, Frankfurt a.M. 1996 (1974), 55f.
} 
Verlauf des infiniten Interpretationsprozesses als gültiger erst noch erweisen muß. Die zunächst nur zeitlich bestimmten Momente der Erfahrung werden in logische Elemente eines Arguments übersetzt, um so eine unbekannte Ursache rekonstruktiv zu erschließen. Der Ausdruck „Retroduktion“ bezieht sich mithin sowohl auf den kausalen Rückschluß von der Wirkung auf die Ursache als auch auf den logischen Rückschluß von der Konklusion auf die Prämissen und auf das Zurückgehen im zeitlichen Sinne. Der Schlußfolgernde projiziert eine logische Form simultan auf die zeitlich aufeinanderfolgenden Elemente seiner inneren Erfahrungswelt und auf die kausal bestimmten Momente der äußeren Erfahrungswelt. Dergestalt wird die Abduktion zu einer innovativen Strategie, die darauf abzielt, Prämissen für gültige Schlußfolgerungen zu finden.

Dabei lassen sich unterschiedliche „Novitätsgrade“ ausmachen, abhängig vom jeweiligen Grad der Determiniertheit durch Vorwissen und konventionaler Codierung: Je nachdem, ob aufgrund von Gewohnheiten quasi-automatisch vom Ergebnis auf die Ursache zurückgeschlossen wird, oder ob situationsabhängige Faktoren darüber entscheiden, welche Erklärung plausibler ist, oder ob man „ex nihilo" Regeln erfinden muß, kann man mit Bonfantini und Proni zwischen „übercodierten “, „untercodierten “ und „kreativen Abduktionen " unterscheiden. ${ }^{55}$ Die übercodierte Abduktion fragt, "was der Fall war" Das heißt, sie entwickelt innerhalb eines durch bereits vorhandene Regeln übercodierten Kontextes als „Schluß auf den Fall“ eine Hypothese. Der untercodierten Abduktion fehlt dagegen eine „passende Regel“, sie ist ein „Schluß aufs Gesetz " und muß sich zwischen mehreren Alternativen die plausiblere auswählen. Im Gegensatz zu unter- und übercodierten Abduktionen geht die kreative Abduktion über die vorgängige, bereits codierte Erfahrung hinaus. Die kreative Abduktion ist Rückschluß auf Gesetz und Fall zugleich, sie ist also die innovative Konstruktion einer Relation zwischen Konklusion und zwei unbekannten Prämissen.

IV.

Nachdem das Abduktionskonzept bislang nur unter einer systematischen, primär epistemologischen Perspektive entfaltet wurde, gilt es nun in einem zweiten Durchgang, die Anschlußfähigkeit der Abduktion an die für das 18 .

55 Massimo Bonfantini, Giampaolo Proni, Raten oder nicht raten?, in: U. Eco und T Sebeok (Hrsg.), Der Zirkel oder im Zeichen der Drei, München 1985, 180-203, hier: 201. Bonfantini und Proni beziehen sich bei ihrer Klassifikation auf Thagards Unterscheidung zwischen „übercodiert" und „untercodiert" (vgl. Paul Thagard, "Semiotics and Hypothetic Inference in C.S. Peirce“, Versus 19/20 [1978], 163-172) und führen als dritten Typ die kreative Abduktion ein. Eco übernimmt diese Einteilung der Abduktionstypen (Vgl. Umberto Eco, „Hörner, Hufe, Sohlen. Einige Hypothesen zu drei Abduktionstypen", in: Eco, Sebeok, Der Zirkel oder im Zeichen der Drei, 288-321, 69).
Jahrhundert zentralen ästhetischen und poetologischen Kategorien der Assoziation, der Einbildungskraft, der Urteilskraft und des Witzes zu erörtern. Das Zusammenspiel dieser vier Kategorien führt zu einem - freilich keineswegs homogenen - Konzept der „ästhetischen Phantasie “, 56 die zugleich auch eine poetologische ist. So bestimmt Breitinger in seiner Critischen Dichtkunst das Dichten als Tätigkeit, „sich in der Phantasie neue Begriffe und Vorstellungen [zu] formieren, deren Originale nicht in der gegenwärtigen Welt der würklichen Dinge, sondern in irgend einem anderen möglichen Welt-Gebäude zu suchen sind ".57 Vergleichsweise enttäuschend ist der Kantische Phantasiebegriff: "Phantasie" spielt in den drei Kritiken Kants als Begriff keine Rolle, abgesehen von einer eher beiläufigen Bemerkung in der Kritik der Urteilskraft, wo vom „Spiel unserer Phantasie“ die Rede ist. ${ }^{58}$ Erst in seiner Anthropologie in pragmatischer Hinsicht erfolgt eine Definition der Phantasie als „Spiel der produktiven Einbildungskraft“, das dem Dichtungsvermögen zugrunde liegt. ${ }^{59}$ Die Einbildungskraft wiederum ist das Vermögen der Vorstellung, „einen Gegenstand auch ohne dessen Gegenwart in der Anschauung vorzustellen “. ${ }^{60}$

Zu fragen bleibt jedoch, ob die Phantasie des Neuen bei Kant lediglich als „Spiel der produktiven Einbildungskraft“ gefaßt werden kann, ob sie nicht vielmehr einem Spiel aller Erkenntnisvermögen geschuldet ist, das von der Urteilskraft vermittelt wird. In der Urteilskraft werden „Verstand und Einbildungskraft im Verhältnisse gegen einander betrachtet “. ${ }^{61} \mathrm{Im}$ Gegensatz zum Verstand als „Vermögen der Regeln“, ist die Urteilskraft das Vermögen, „unter Regeln zu subsumieren, d.i. zu unterscheiden, ob etwas unter einer gegebenen Regel (casus datae legis) stehe, oder nicht “ ${ }^{62}$ Eine Möglichkeit, Verstand und Einbildungskraft ins Verhältnis zu setzen, ist das Aufstellen von Hypothesen. Sobald man nämlich die Einbildungskraft mit einem möglichen (verstandesmä-

56 Vgl. Christoph Unger, Die ästhetische Phantasie. Begriffsgeschichte, Diskurs, Funktion, Transformation. Studien zur Poetologie Jean Pauls und Johann Wolfgang Goethes, Frankfurt a.M., Berlin, Bern, New York, Paris, Wien 1996, $27 \mathrm{ff}$.

57 Johann Jakob Breitinger, Critische Dichtkunst, Stuttgart 1966 (1740), 60.

${ }_{8}$ Immanuel Kant, Kritik der Urteilskraft, in: Werkausgabe (Anm.13), X, 262.

59 Kant (Anm. 13), 483. Dabei macht Kant die interessante Feststellung, daß nicht nur wir mit der Einbildungskraft spielen, sondern „die Einbildungskraft (als Phantasie) spielt eben so oft und bisweilen sehr ungelegen auch mit uns" (476).

${ }^{60}$ Immanuel Kant, Kritik der reinen Vernunft, in: Werkausgabe (Anm. 13), III/IV, 148.

${ }^{61}$ Kant (Anm. 58), 36.

62 Kant (Anm. 60), $184 \mathrm{ff}$. Vgl. hierzu auch Schönrich, der das Dilemma regelgeleiteten Handelns betont, daß jede Anwendungsregel wiederum eine Regel braucht, „die ihrerseits die Anwendung dieser Anwendungsregel regelt und so ad infinitum “, dieses Dilemma wird, wie Schönrich feststellt, von Kant „durch die Einführung der Urteilskraft als einer Regelkompetenz aufgelöst, die den Hiatus zwischen Regel und Fall überbrückt“. (Gerhard Schönrich, Zeichenhandeln - Untersuchungen zum Begriff einer semiotischen Vernunft im Ausgang von Ch.S.Peirce, Frankfurt a.M. 1990, 391f.) 
Bigen) Erklärungsgrund verknüpft, entsteht eine Hypothese. ${ }^{63}$ Damit sind wir wieder bei der Abduktion: Die unterschiedlichen Formen, die die Abduktion annehmen kann, möchte ich als unterschiedliche Spiele der Erkenntnisvermögen interpretieren.

Die bestimmende Urteilskraft operiert analog zum übercodierten abduktiven $S c h l u \beta$ auf einen hypothetisch angenommenen Fall, wobei die Regel vorausgesetzt wird: „Ist das Allgemeine (die Regel, das Prinzip, das Gesetz) gegeben, so ist die Urteilskraft [...] bestimmend". ${ }^{64}$ Dem untercodierten abduktiven Schluß auf die Regel entspricht die reflektierende Urteilskraft. Dieser ist "das Besondere gegeben, wozu sie das Allgemeine finden soll ", 65 wobei entweder zwischen mehreren möglichen Regeln eine plausible ausgewählt oder aber eine Regel erfunden werden muß. Während die reflektierende Urteilskraft ästhetisch sein kann, ${ }^{66}$ versucht die bestimmende Urteilskraft durch ein logisches Urteil, einen „zu Grunde liegenden Begriff durch eine gegebene empirische Vorstellung zu bestimmen “. ${ }^{67}$ Die reflektierende Urteilskraft stiftet dagegen neue, sinnfällige Zusammenhänge: „Reflektieren (Überlegen) aber ist: gegebene Vorstellungen entweder mit andern, oder mit seinem Erkenntnisvermögen, in Beziehung auf einen dadurch möglichen Begriff, zu vergleichen und zusammen zu halten " ${ }^{68}$ Die kreative Abduktion schließlich initiiert ein Spiel der Erkenntnisvermögen, das zur „Bedingung der Möglichkeit“ jeder Phantasie des Neuen wird. Die kreative Abduktion setzt „eine freie und uneingeschränkte Bewegung " 69 im Spannungsfeld der Erkenntnisvermögen Verstand, Einbildungskraft und Urteilskraft in Gang, die zwischen Instinkt und Ingenium oszilliert.

In seiner Anthropologie in pragmatischer Hinsicht präzisiert Kant den Unterschied von der reflektierenden Urteilskraft und der logisch bestimmenden Urteilskraft, wobei er die regelfindende Urteilskraft als „Ingenium“ und als "Witz" bezeichnet: Der Begriff Ingenium umfaßt dabei sowohl Scharfsinn und Erkenntnisfähigkeit als auch Erfindungskraft und Phantasie: ${ }^{70}$ "So wie das Vermögen, zum Allgemeinen (der Regel) das Besondere auszufinden, Urteilskraft, so ist dasjenige: zum Besonderen das Allgemeine auszudenken, der Witz (ingenium) ${ }^{\text {“ }}{ }^{71}$ Kant setzt hier Witz qua Ingenium mit reflektierender Urteilskraft gleich, während Urteilskraft die verkürzte Bezeichnung der bestimmenden Urteilskraft wird. Mit Blick auf ihre jeweilige spezifische Vermittlungslei-

${ }^{63}$ Vgl. Kant (Anm. 60), 652f.

64 Kant (Anm. 58), 87.

65 Kant (Anm. 58), 87.

${ }_{66}^{6}$ Kant (Anm. 58), 37.

${ }^{67}$ Kant (Anm. 58), 24.

68 Kant (Anm. 58), 87.

${ }^{69}$ Kant (Anm. 60), 611

${ }_{70}$ Vgl. Waltraud Wiethölter, Witzige Illumination. Studien zur Ästhetik Jean Pauls, Tübingen 1979, 3.

${ }^{71}$ Kant (Anm. 13), 511 stung zwischen Verstand und Vernunft schreibt Kant: „Witz hascht nach Einfällen; Urteilskraft strebt nach Einsichten " ${ }^{72}$ Die Vermittlungsinstanz zwischen dem Haschen nach Einfällen und dem Streben nach Einsichten ist der Mutterwitz, „dessen Mangel keine Schule ersetzen kann“, da es sich um eine "Gabe der Natur" handelt. ${ }^{73}$

Für Peirce hat diese „Gabe der Natur " die Form des „Instinkts", der sich im angeborenen Vermögen, richtig zu raten, äußert. ${ }^{74}$ Dieser „Guessing Instinct ${ }^{\text {“ }}$ entwickelt sich aus einer „natürlichen Affinität" zwischen dem Geist des Denkenden und seiner natürlichen und kulturellen Lebenswelt, deren "geheime Gesetze" er verinnerlicht und adaptiert hat. Der "Guessing Instinct" rekurriert auf ein, wie Peirce es nennt, „divinatorisches Vermögen“ („faculty of divining "),${ }^{75}$ mit dessen Hilfe es dem Menschen in the long run gelingt, die "geheimen Prinzipien " des Universums zu entschlüsseln und zu rationalisieren. Da der Mensch Teil dieses Universums ist und sich „unter dem Einfluß dieser Gesetze" entwickelt hat, kann man davon ausgehen, "that he should have a natural light, or light of nature, or instinctive insight, or genius, tending to make him guess those laws aright, or nearly aright ${ }^{4} .{ }^{76}$ Diese kongeniale, instinktive Einsicht in die Zusammenhänge, deren Teil wir sind, bezeichnet Peirce, genau wie Kant, als „unconscious or semi-conscious irreflective judgements of mother-wit ". ${ }^{77}$ Dabei erweist sich der „Guessing Instinct" als eine Art „hermeneutischer Spürsinn fürs Relevante", der durch die Interferenz von Instinkt und ökonomischer Klugheitsstrategie zum „divinatorischen Verfahren“ wird. ${ }^{78}$

\footnotetext{
${ }^{72}$ Kant (Anm. 13), 539.

${ }^{73}$ Kant (Anm. 60), 184ff.

74 Vgl. Peirce (Anm. 44), 282.

75 Dort heißt es: „man divines somerhing of the secret principles of the universe because his mind has developed as a part of the universe and under the influence of these same secret principles“ (Peirce [Anm. 5], 7.46).

76 Peirce (Anm. 5), 5.604.

77 Peirce (Anm. 5), 6.623.

78 Der Begriff des „divinatorischen Erratens" spielt in Schleiermachers Hermeneutik eine zentrale Rolle. Der Prozeß des Verstehens erfordert nämlich das Wissen um die geschichtliche Situation, die Anwendung der „allgemeinen Gesetze der Ordnung im Denken“, wobei die Konjektur, ebenso wie das „Erraten“, im Zentrum des „divinatorischen Verfahrens" steht (F.D.E. Schleiermacher, Hermeneutik und Kritik, hrsg. Manfred Frank, Frankfurt a.M. 1990, 210 und 264). Roland Daube-Schackat hat in seinem Aufsatz „Schleiermachers Divinationstheorem und Peirce's Theorie der Abduktion “ das Verhältnis beider Konzepte untersucht. Nach Schackat teilt Schleiermachers Begriff der Divination als konjekturale Erkenntnisfähigkeit mit der Abduktion ein wesentliches Merkmal, nämlich das ihrer Fallibilität (vgl. Roland Daube-Schackat, „Schleiermachers Divinationstheorem und Peirce's Theorie der Abduktion “, in: K.V. Selge [Hrsg.], Internationaler Schleiermacherkongreß Berlin 1984, Berlin und New York 1985, 263-278, hier: 273).
} 
Mit Blick auf Peirce und Kant könnte man sagen: die auf Einfälle und Einsichten abzielende Phantasie des Neuen pendelt zwischen instinktivem Mutterwitz, der sich unter den Gesetzen der Natur entwickelt, und dem witzigen Ingenium, durch welches die Natur der Kunst die Regel gibt. Genie wird in der Kritik der Urteilskraft bekanntlich als Talent definiert, „dasjenige, wozu sich keine bestimmte Regel geben läßt, hervorzubringen “, woraus folgt, „daß Originalität seine erste Eigenschaft sein müsse“. ${ }^{79}$ Das Genie, das „als Natur “ die Regel gibt, ist dabei zwar „Urheber eines Produkts, welches er seinem Genie verdankt“, aber es weiß selbst nicht, "wie sich in ihm die Ideen dazu herbei finden ", zumal es nicht in seiner Gewalt steht, „dergleichen nach Belieben oder planmäßig auszudenken “ ${ }^{80}$ Das Feld des Genies ist die Einbildungskraft, und da diese "weniger als andere Vermögen unter dern Zwange der Regeln steht", ist sie "der Originalität desto fähiger" ${ }^{81}$

Gerade was die Freiheit vom Zwang der Regeln betrifft kann Kants Geniekonzept als transzendentale Unabhängigkeitserklärung von jenen drei Assoziationsprinzipien - „Resemblance, Contiguity in time or place, and Cause and Effect" - gewertet werden, die nach Hume einen "equal influence on all mankind" haben. ${ }^{82}$ Selbst in unseren „wildest and most wandering reveries, nay in our very dreams", so Hume, könnten wir beobachten, daß sich unsere Vorstellungen (imaginations) nicht völlig abenteuerlich bewegen, sondern, daß es zwischen den verschiedenen Vorstellungen immer wieder ähnliche Verknüpfungen (connections) und Übergänge (transitions) gibt. ${ }^{83}$ Während die Verknüpfungsformen zwischen den Ideen durch die "principles of association" festgelegt werden, sind die Verknüpfungsmöglichkeiten im Rahmen der Einbildungskraft völlig frei:

„Nothing is more free than the imagination of man; and though it cannot exceed that original stock of ideas furnished by the internal and external senses, it has unlimited power of mixing, compounding, separating, and dividing these ideas, in all the varieties of fiction and vision ". ${ }^{84}$

Ganz ähnlich heißt es im Artikel ,imagination“ in der Encyclopédie, das Herstellen neuer Zusammenhänge obliege einer imagination active, „qui arrange ces images reçues, \& les combine en mille manieres". ${ }^{85}$ Diese Möglich-

\footnotetext{
79 Kant (Anm. 58), 242.

${ }^{80}$ Kant (Anm. 58), 243.

81 Kant (Anm. 13), 544

82 David Hume, Enquiries concerning the buman understanding and concerning the principles of morals, Reprinted from the edition of 1777 by L.A. Selby-Bigge, Oxford 1957,$48 ; \$ 39$.

83 Hume (Anm. 82), 23; $\$ 18$.

${ }^{84}$ Hume (Anm. 82), 47; \$39.

${ }^{85}$ Jean Le Rond D'Alembert, Denis Diderot (Hrsg.), Encyclopédie, Paris 1765, VIII, Artikel „Imagination", 561 .
}

keit zum Arrangieren, Re-Arrangieren und Neu-Arrangieren stellt für die Assoziationstheorie die Voraussetzung für die Phantasie des Neuen in Form der imagination d'invention ${ }^{86}$ dar.

Kant hält diese Auffassung für unzureichend. Im Rahmen seiner Kritik an Humes empiristischer Assoziationstheorie führt er zunächst die Unterscheidung zwischen reproduktiver und produktiver Einbildungskraft ein. Während die Synthesis der reproduktiven Einbildungskraft „lediglich empirischen Gesetzen, nämlich denen der Assoziation, unterworfen ist", zeichnet sich die produktive Einbildungskraft durch ${ }_{n}$ Spontaneität ${ }^{487}$ aus, also durch das Vermögen, nicht nur Vorstellungen zu empfangen, sondern mit Hilfe dieser Vorstellungen Gegenstände zu erkennen. ${ }^{88}$ Die "produktive Einbildungskraft" ist jedoch nur ein Aspekt der imagination d'invention. Die Phantasie des Neuen verdankt sich der spielerischen Vermittlungsleistung der Urteilskraft, die Verstand und Einbildungskraft in ein „glückliches Verhältnis“ bringt. So besteht die spezifische Leistung der reflektierenden Urteilskraft als witziges Ingenium darin, "heterogene Vorstellungen“ zu „paaren“, „die oft nach dem Gesetze der Einbildungskraft (der Assoziation) weit auseinander liegen “. ${ }^{89}$ Das Genie transzendiert jedoch auch noch diese verknüpfende Leistung des witzigen Ingeniums, denn es zeichnet sich durch das Vermögen aus, „das schnell vorübergehende Spiel der Einbildungskraft aufzufassen, und in einen Begriff (der eben darum original ist und zugleich eine neue Regel eröffnet, die aus keinen vorhergehenden Prinzipien oder Beispielen hat gefolgert werden können) zu vereinigen, der sich ohne Zwang der Regeln mitteilen läßt ${ }^{\prime \prime} .{ }^{90}$ Das wahre Genie zeigt sich also darin, daß es das "glückliche Verhältnis" von Verstand und Einbildungskraft in einem neuen, nicht nur originalen, sondern originellen Token zum Ausdruck bringt.

\section{V.}

Kants Bestimmung der reflektierenden Urteilskraft als Witz betrifft nicht nur deren Vermögen, Regeln zu finden, sondern auch Ähnlichkeiten. Für die Erkenntnistheorie des 18. Jahrhunderts ist der Witz als „Ähnlichkeitsfinder“ Stimulus jeder neuen, synthetischen und originalen Erkenntnis. Diese Auffassung bringt Lichtenberg auf den Punkt, wenn er feststellt, daß der Mensch ohne Witz „gar nichts“ sei, „denn Ähnlichkeit in den Umständen ist ja alles, was uns zur wissenschaftlichen Erkenntnis bringt, wir können ja bloß nach Ähnlichkei-

86 D'Alembert, Diderot (Anm. 85), 561.

87 Kant (Anm.60), 149.

$88 \mathrm{Vgl}$. Kant (Anm. 60), 97.

89 Kant (Anm.13), 537f.

90 Kant (Anm. 58), 254. 
ten ordnen und behalten". ${ }^{91}$ Im Gegensatz zu Lichtenberg und Kant, die die epistemologische Funktion des Witzes betonen, fokussiert die romantische Theorie des Witzes dessen universale, poetische Funktion. Der Witz wird zum Prinzip einer Universalphilosophie erklärt, die zugleich Universalpoesie ist. $\mathrm{Er}$ wirdzur „logischen Chemie“ einer Philosophie, die sich als „Wissenschaft aller sich ewig mischenden und wieder trennenden Wissenschaften " versteht. ${ }^{92} \mathrm{Da}$ bei ist der „chemische " Witz Ausdruck des „organischen " Genies: beiden eignet Freiheit, beide bringen neue Ideen ins Spiel. ${ }^{93}$ In die gleiche Richtung wie Schlegel weist Jean Paul, wenn er schreibt: „Freiheit gibt Witz und Witz gibt Freiheit". ${ }^{94}$ Das kreative Potential des Witzes liegt in der spielerischen Bewegung gedanklicher Freiheit begründet:

„Aber, Himmel, welche Spiele könnten wir gewinnen, wenn wir mit unsern einsiedlerischen Ideen rochieren könnten! Zu neuen Ideen gehören durchaus freie; zu diesen wieder gleiche; und nur der Witz gibt uns Freiheit, indem er Gleichheit vorher gibt, er ist für den Geist, was für die Scheidekunst Feuer und Wasser ist, Chemica non agunt nisi soluta (d.h. nur die Flüssigkeit gibt die Freiheit zu neuer Gestaltung - oder: nur entbundne Körper schaffen neue) “.95

Sowohl von Schlegel als auch von Jean Paul wird die „logische Chemie" des Witzes als Möglichkeit gefaßt, Ideen in Bewegung zu halten, damit diese neue Verbindungen eingehen und neue Reaktionen auslösen können: „Witz ist eine Explosion von gebundenem Geist “, ${ }^{96}$ lautet die Formel. Zugleich bleibt der Witz „logische Geselligkeit “, ${ }^{97}$ auch wenn diese Geselligkeit die gültigen intersubjektiven Konventionen übertreten muß, um neue Verbindungen einzugehen. Man denke an Jean Pauls berühmte Definition des Witzes als ,verkleideter Priester, der jedes Paar kopuliert ". ${ }^{98}$ Sie impliziert, daß zwei heterogene Vorstellungen gepaart werden, wobei Jean Paul am Assoziationsbegriff einen innovativen Tausch der Bedeutungsnuancen vornimmt - die assoziative Verknüpfung der Ideen wird als soziale Vereinigung gedeutet. In der Encyclopédie heißt es unter dem Stichwort „Association“: „Le plus stable de toutes les associa-

${ }_{91}^{1}$ Lichtenberg (Anm. 6), 167.

${ }_{92}$ Friedrich Schlegel, Athenäums-Fragmente, in: Kritische Friedrich-Schlegel-Ausgabe, 1. Abteilung, hrsg. Ernst Behler, Hans Eichner, München, Paderborn, Wien 1967, II, 200, vgl. hierzu auch Eckhart Oehlenschläger, Närrische Phantasie. Zum metaphorischen Prozeß bei Jean Paul, Tübingen 1980, $12 \mathrm{f}$.

${ }_{93}$ Schlegel, Kritische Fragmente (Anm. 92), 148.

94 Jean Paul, Vorschule der Ästhetik, in: Werke, hrsg. Norbert Miller, München 1980, IX, 201.

${ }_{95}$ Jean Paul (Anm. 94), 200.

96 Schlegel (Anm. 93), 158.

${ }_{98}^{97}$ Schlegel (Anm. 93), 154.

98 Jean Paul (Anm. 94), 173. Vgl. hierzu auch: Bettine Menke, „Jean Pauls Witz. Kraft und liormel “, DVis 76 (2002), 201-213, hier: 202. tions est celle qui se fait par le marriage". ${ }^{99}$ Die Heirat erscheint als feste assoziative Kopplung. Das Pendant dieser festen assoziativen Kopplung in der sozialen Welt sind die Kopula in der sprachlichen Welt. Die „Trauformel“, mit der der verkleidete Priester die witzige Vereinigung stiftet, ist die "SprachGleichsetzung im Prädikat “ ${ }^{100}$ seine Verkleidung ist die Homonymie, nämlich daß „für beide ein Zeichen des Prädikats gefunden wird “. ${ }^{101}$ Das witzige Genie im Sinne Jean Pauls gibt sich nicht nur „als Natur“ die Regel, sondern es zeichnet sich dadurch aus, daß es die Regeln der Kultur - etwa die illokutionären Gelingensbedingungen des Heiratens - phantasievoll unterläuft. Der Witz folgt dabei der gleichen Maxime wie die Phantasie: Ersterer kopuliert jedes Paar, letztere "macht alle Teile zu Ganzen". ${ }^{102}$

Vergleicht man die romantische Witz- und Geniekonzeption mit der Kantischen, so lassen sich zwei grundlegende Unterschiede ausmachen, die für die Frage, ob es eine den Wissenschaften und den Künsten gemeinsame Phantasie des Neuen gibt, relevant sind. Erstens: Während Kant das regelerfindende Talent des Genies darauf beschränkt, „daß die Natur durch das Genie nicht der Wissenschaft, sondern der Kunst die Regel vorschreibe “, ${ }^{103}$ nivellieren Jean Paul und Schlegel die Differenz zwischen dem philosophischen und dem poetischen Genius: „Die erfindenden Philosophen waren alle dichterisch “. ${ }^{104}$ Schlegel geht sogar noch einen Schritt weiter, wenn er in den Kritischen Fragmenten eine Demokratisierung des Geniegedankens fordert: „Man soll von jedermann Genie fordern, aber ohne es zu erwarten. Ein Kantianer würde dies den kategorischen Imperativ der Genialität nennen ". ${ }^{105}$ Zweitens: Die romantische Witzkonzeption betont, daß der Witz nicht mehr nur Finder, sondern Erfinder von Ähnlichkeiten ist. ${ }^{106}$ So schreibt Jean Paul: „Der Witz allein daher erfindet, und

\footnotetext{
99 Stichwort „Association“, in: Encyclopédie, hrsg. Jean Le Rond D'Alembert, Denis Diderot, Paris 1751, I, 771.

100 Jean Paul (Anm. 94), $173 \mathrm{ff}$.

101 Ebd. Zugleich unterhält der Hinweis auf den ,verkleideten Priester" eine unterirdische Verbindung zu Kants Begriff der Subreption, den er in der Kritik der reinen Vernunft ebenso wie in der Kritik der Urteilskraft für eine bestimmte Form der Verwechslung verwendet: Der Begriff der Subreption bezeichnet sowohl einen bewußt fehlerhaften Beweisschluß, der sich auf Voraussetzungen stützt, die nicht auf Tatsachen beruhen, als auch das unrechtmäßige Erlangen eines Erfolges durch Verschleierung des wahren Sachverhalts im juristischen Kontext. Im kanonischen Recht steht der Terminus Subreption für "die betrügerische Erschleichung eines priesterlichen Amtes" (Vgl. David E. Wellbery, „Die Enden des Menschen. Anthropologie und Einbildungskraft im Bildungsroman", in: Karlheinz Stierle, Rainer Warning [Hrsg.], Das Ende. Figuren einer Denkform, München 1996, 607 [fn]).

102 Jean Paul (Anm.94), 47.

103 Kant (Anm. 58), 243.

104 Jean Paul (Anm.94), Fn. 56.

105 Schlegel (Anm. 93), 148

106 Jean Paul bemängelt an der alten Definition des Witzes als eines Vermögenis, ent-
} 
zwar unvermittelt, daher nennt ihn Schlegel mit Recht fragmentarische Genialität". 107

Im Gegensatz zum „scharfsinnigen Witz“, der zwischen „den gefundenen Verhältnissen kommensurabler und ähnlicher Größen wieder Verhältnisse findet und unterscheidet“, stellt der ästhetische Witz „Verhältnisse inkommensur abler (unmeßbarer) Größen“ her, „d.h. die Ähnlichkeiten zwischen Körperund Geisteswelt“, wobei diese Ähnlichkeit durch einen „Instinkt der Natur“ erzwungen wird, mithin „offen und stets auf einmal da[liegt]“. ${ }^{108}$ Diese Form des Witzes wird zu einem Licht, das "aus der Wolke selber fährt " ${ }^{\circ}, 09$ ist also ein Gedankenblitz: „Den Witz und den komischen Einfall erschöpft und entladet, wie den zickzackigen Blitz, der erste Schlag “. ${ }^{110}$ Auch Schlegel spricht vom „geistige[n] Blitz des Verständnisses, der aufsteigt, wenn in der unmittelbaren Berührung der Sinn aufgefaßt wird “. Dieser Gedankenblitz ist als „augenblickliche Schöpfung des Geistes, sozusagen eine Schöpfung aus nichts“. Dabei ist dieses schöpferische Berühren des Geistes für Schlegel nichts anderes „als ein Dichten". ${ }^{111}$

\section{VI.}

Die Rede vom „Gedankenblitz“ hat, wie die Blitzmetaphorik überhaupt, sowohl für die romantischen als auch für die nachromantischen Theorien der künstlerischen Produktivität zentrale Bedeutung. So vergleicht Konstantin Paustowski den Einfall mit einem Blitz, der sich in einer angestauten, gewittrigen Atmosphäre entlädt:

„Der Einfall kommt ebenso wie der Blitz in einem mit Gedanken, mit Gefühlen und Gedächtnisnotizen gesättigten Bewußtsein eines Menschen zustande.

fernte Ähnlichkeiten zu finden, daß hier „weder, entfernte ${ }^{\star}$ bestimmt, noch, Ähnlichkeit wahr" sei. Der Begriff der "fernen Ähnlichkeit" sei ein Widerspruch in sich, da er soviel wie „Unähnlichkeit" bedeute, während der zweite Teil der Definition den Witz als „Finder der Ähnlichkeit“ ganz vom Scharfsinn, als „Finder der Unähnlichkeit “ trennt. Jean Paul betont dagegen, daß nicht nur „die Vergleichungen des Witzes oft Unähnlichkeiten“ ergeben, sondern auch „die Vergleichungen des Scharfsinnes“ oft Ännlichkeiten hervorbringen (Jean Paul [Anm. 94], 169f.).

107 Jean Paul (Anm.94), 171.

108 Jean Paul (Anm.94), 172. Interessant ist hier Jean Pauls Bezugnahme auf den Instinkt, der dem Auffinden von Ähnlichkeiten zugrunde liegt. An anderer Stelle schreibt er in völliger Übereinstimmung mit der Peirceschen Bestimmung des Instinktes: „Der Instinkt oder Trieb ist ein Sinn der Zukunft [...]. Er bedeutet und enthält seinen Gegenstand ebenso wie die Wirkung die Ursache “ $(60)$.

109 Jean Paul (Anm. 94), 172.

110 Jean Paul (Anm. 94), 470.

111 Friedrich Schlegel, Philosophische Vorlesungen (1800-1807), in: Kritische Friedrich-Schlegel-Ausgabe, hrsg. Jean Jacques Anstett, Ernst Behler, München, Paderborn, Wien 1964, XIII, 374 f.
Das sammelt sich alles nach und nach, langsam, bis es jenen Grad von Spannung erreicht, der unbedingt eine Entladung verlangt. Dann bringt diese gedrängte und noch etwas chaotische Welt einen Blitz - den Einfall - hervor“. ${ }^{112}$

Der Blitz stellt aber auch eine systematische Beziehung zwischen „Witz “ und "Abduktion“ her: Die abduktive Vermutung verdankt sich nämlich einem blitzartigen Akt der Einsicht: „The abductive suggestion comes to us like a flash“. ${ }^{113}$ Die Abduktion ist ein Gedankenblitz, dessen Pointe in der Idee „of putting together what we had never before dreamed of putting together" besteht. ${ }^{114}$ Zwar waren die verschiedenen Elemente der Hypothese zuvor in unserem Geist, aber erst die konjekturale Idee, diese Elemente „zusammenzuwerfen “, läßt blitzartig eine neue Vermutung entstehen - „flashes the new suggestion before our contemplation “. ${ }^{115}$ Den abduktiven Gedankenblitz kann man mit Lorenz als „Fulguration“ begreifen, ${ }^{116}$ als Vorgang, bei dem auf einmal etwas noch nie Dagewesenes entsteht.

Die Idee, zwei Elemente, die gemeinsam assoziiert werden, auch konjektural in eine logische Relation zueinander zu stellen, vollzieht sich im Modus ästhetischer Plötzlichkeit. Genau wie die witzige Ideen-Rochade Jean Pauls stellt die Abduktion als „Kurzschluß“ neue Zusammenhänge her. Witz und Abduktion sind „rekontextualisierende Operationen “ der Phantasie. Diese Rekontextualisierungsbewegung nimmt einen "innovativen Tausch“ vor, der entweder als Rochade von Prämissen oder aber als Aufpfropfung (greffe) im Sinne Derridas gedeutet werden kann. Das heißt, Witz und Abduktion beruhen auf der nicht zu sättigenden Möglichkeit, Gedanken-Zeichen aus Kontexten herauszulösen und auf neue Kontexte aufzupfropfen. ${ }^{117}$ Die Bedingung für die abduktive ebenso wie für die witzige Entladung ist eine Mischung aus angespannter Erwartung einerseits und schlagartiger Verknüpfung von ungebundenen Elementen - „freien Ideen“ im Sinne Jean Pauls - andererseits. Solch ein „indeterminierter Zustand“ ist nach Bohrer konstitutiv für den „kreativen Augenblick“, der eine plötzliche, schlagartige Veränderung des Bewußtseins bewirkt: „Eine solche Veränderung ist eine Entscheidung, nennen wir sie nun ,existentiell

112 Konstantin Paustowski, „Der Blitz“, in: M. Curtius (Hrsg.), Seminar: Theorien der künstlerischen Produktivität, Frankfurt a.M. 1976, 280 f.

113 Peirce (Anm. 5), 5.181.

${ }^{114}$ Peirce (Anm. 5), S.181.

115 Peirce (Anm. 5), 5.181.

116 Der Begriff der „Fulguration“ wird von Konrad Lorenz eingeführt, um „das Auftreten von total Neuem, von nie Dagewesenem “ zu bezeichnen. Vgl. Karl R. Popper und Konrad Lorenz, Die Zukunft ist offen. Das Altenberger Gespräch, hrsg. F. Kreuzer, München, Zürich, 6. Aufl. 1994, $15 \mathrm{f}$.

117 Vgl. Jacques Derrida, „Signature événement contexte ${ }^{4}$, in: ders., Marges de la philosophie, Paris 1972, 365-393, hier: 381. 
oder den Prozeß intuitiver Erleuchtung “. ${ }^{118}$ Die Blitzartigkeit des Einfalls impliziert, daß die Gedanken durch eine innere logische Chemie - sei es in Form einer instinktiven, assoziativen Suggestion, sei es in Form eines freien, witzigen Kurzschlusses - miteinander verknüpft werden. Die Rede vom abduktiven Gedankenblitz verweist aber auch auf den Kairos-Begriff als Moment der Offenbarung und des "großen Augenblicks“. ${ }^{119}$ Dieser wird zugleich als étonnement und als überraschende, schlagartige Umkehr aufgefaßt. ${ }^{120}$

Für Peirce ist die Motivation für die blitzartige, abduktive Synthese ein plötzlich entfachtes, unsystematisches und subjektives Erkenntnisinteresse in Form eines unkontrollierten, vergnüglichen Gedankenspiels: „But when in the uncontrolled play of that part of thought, an interesting combination occurs, its subjective intensity increases for a short time with great rapidity ". ${ }^{121}$ Bemerkenswerterweise ist die Kantische Beschreibung der durch den Witz ausgelösten gedanklichen Bewegung beinahe gleichlautend. Nach Kant erregt der Witz Interesse, „und zwar durch Ideen “. ${ }^{22}$ Diese Ideen setzen „die Einbildungskraft in Bewegung, welche für dergleichen Begriffe einen großen Spielraum vor sich sieht ". ${ }^{123}$ Bei Peirce wird diese Bewegung durch eine Ideenkombination ausgelöst, die die Aufmerksamkeit steigert: entweder durch Überraschung und Schock oder durch Suggestion und Affektion. ${ }^{124}$ Diese subjektive Intensität des „direkten Bewußtseins“ besteht in der Entdeckung eines neuen, kohärenten Zusammenhangs aufgrund einer interessanten Ähnlichkeitsrelation. Dabei erweist sich die Abduktion als "Logic of Discovery“.

Die Abduktion repräsentiert als Originary Argument in ihren Prämissen Fakten, "which present a similarity to the fact stated in the Conclusion". ${ }^{125}$ Das heißt, zwischen dem propositionalen Gehalt der Prämissen und dem propositionalen Gehalt der Konklusion besteht eine Ähnlichkeitsassoziation, die einem Analogieschluß Vorschub leistet: "we are not led to assert the Conclusion positively but are only inclined toward admitting it as representing a fact of which the facts of the Premiss constitute an Icon ". ${ }^{26}$ Wir betrachten mithin die Konklusion „als ob“ sie eine Tatsache repräsentiert, die zu der in der Prä-

${ }^{118}$ Karl-Heinz Bohrer, Plötzlichkeit - Zum Augenblick des ästhetischen Scheins, Frankfurt a.M. 1981, 82.

$119 \mathrm{Vgl}$. Klaus Ley, „Kunst und Kairos - Zur Konstitution der wirkungsästhetischen Kategorie von Gegenwärtigkeit in der Literatur “, Poetica 17 (1985), 46-82, hier: 46.

120 „Im ,étonnement', im Augenblick der höchsten Einsicht, der ganzen , Wahrheit kommt die radikale Betroffenheit, die in der Imagination durchlebt wird, zu voller Wirkung" (Ley [Anm. 119], 48).

121 Peirce (Anm. 5), 7.555.

122 Kant (Anm. 13), 544.

123 Kant (Anm. 13), 544.

$124 \mathrm{Vgl}$. Peirce (Anm. 5), 7.397.

125 Peirce (Anm. 5), 2.96.

126 Peirce (Anm. 5), 2.96. misse repräsentierten Tatsache in einer ikonischen Ähnlichkeitsrelation steht. Der abduktive Einfall kommt durch eine überraschende Ähnlichkeitsassoziation zustande, eine Ähnlichkeitsassoziation also, die nicht einfach durch ein explizit gegebenes Prädikat zustandekommt, sondern durch ein implizit präsupponiertes.

Peirce nennt als Beispiel Keplers „revolutionäre“ Theorie über die Umlaufbahn des Mars. Nachdem Kepler lange Zeit vergeblich über die verschiedenen Möglichkeiten nachgedacht hatte, die Daten mit der präfigurierten Vorstellung einer runden Umlaufbahn in Einklang zu bringen, nahm er versuchsweise eine elliptische Form an, das heißt, er ersetzte die major-Prämisse seiner Argumentation. ${ }^{127}$ Tatsächlich paßten die aus Beobachtung resultierenden Daten in ein Diagramm, das eine elliptische Form hatte. Mithin bestand eine ikonisch-diagrammatische Relation zwischen der hypothetischen major-Prämisse und der Konklusion, nämlich den beobachteten Daten.

Die der ikonischen Bezeichnungsweise zugrundeliegende Anziehungskraft des Ähnlichen ruft eine „sympathetische“ Reaktion hervor, die auf den Interpreten "suggestiv“ wirkt. ${ }^{128}$ Die Suggestion verstärkt entweder ein bestehendes Kontiguitätsverhältnis, oder sie stellt einen neuen analogischen Zusammenhang durch eine willkürlich auferlegte Ähnlichkeitsbeziehung her. ${ }^{129}$ Die bei der Abduktion zwischen Konklusion und Prämisse bestehende „ikonische Suggestivkraft“ stellt den „witzigen Stimulus“ für das Erkenntnisvermögen dar. Aus dieser suggestiven Anregung des Erkenntnisvermögens kann „neue Erkenntnis" erwachsen, sie kann aber auch ein reines Spiel der Phantasie bleiben. In beiden Fällen handelt es sich um Gedankenspiele. In beiden Fällen läßt sich die abduktive "creation of hypothesis" als "grand work of poietic genius" bezeichnen. ${ }^{130}$

\section{VII.}

In seinem Essay Hume on Miracles beschreibt Peirce das Gedankenspiel als „certain agreeable occupation of mind“, das keinen anderen Zweck verfolgt,

127 Vgl. hierzu auch Alan G. Gross, The Rhetoric of Science, Cambridge 1990.

$128 \mathrm{Vgl}$. Peirce (Anm. 5), 8.370ff.

$129 \mathrm{Vgl}$. Roman Jakobson, „Die Sprache in ihrem Verhältnis zu anderen Kommunikationssystemen“, in: Eugenio Coseriu (Hrsg.), Form und Sinn, München 1974, 162-175, hier: 171.

${ }_{130}$ Peirce (Anm. 5), 4.238. Siehe hierzu auch Michael Cabot Haley, The Semiosis of Poetic Metaphor, Bloomington 1988, der den metaphorischen Erkenntniszuwachs mit einer wissenschaftlichen Entdeckung vergleicht: „My point is simply that the same reality can be the object of either kind of hypothesis, scientific or metaphorical, and that what makes the scientific hypothesis prove successful is an indispensable part (though not the whole) of what makes a metaphorical connection , poetic ${ }^{2}-$ its approximation to reality. " (52) 
als „casting aside all serious purpose“. ${ }^{131}$ Dieser Geisteszustand kann entweder die Form der reverie, also des Tagtraums, oder des Musement, also der Grübelei, annehmen. In beiden Fällen handelt es sich um Pure Play, das nur eine Regel kennt: „this very law of liberty“.132 Dieses Spiel kann als ästhetische Kontemplation ("aesthetic contemplation“) in Erscheinung treten oder als imaginäres „castle building“, als Spekulation über wie auch immer geartete Verknüpfungen („connection“) zwischen den drei „Universen“ Gedankenwelt, Zeichenwelt, Außenwelt bzw. als Spekulation über die möglichen Ursachen dieser Verknüpfungen. Eben diese "speculation concerning its cause“ zeichnet das Musement, also das grübelnde Gedankenspiel, aus. ${ }^{133}$

Die Peircesche Formulierung, daß das „reine Spiel“ keine andere Regeln kennt als das „Gesetz der Freiheit“, geht auf Kant und Schiller zurück. ${ }^{134}$ Bei Kant ist es das „freie Spiel der Einbildungskraft“, ${ }^{135}$ die im „freien Spiel der Ideen [...] und ohne bestimmten Zweck die ästhetische Urteilskraft [beschäftigt] “. ${ }^{136}$ Schiller betont, daß das Spiel als „freie Bewegung “ der Einbildungskraft und als „ungezwungene Folge von Bildern“ ohne jeden „äußeren sinnlichen Zwang “ entsteht und sich „ihrer Eigenmacht und Fessellosigkeit" freut. Aus dem "Spiel der freien Ideenfolge" macht die Einbildungskraft „in dem Versuch einer freien Form den Sprung zum ästhetischen Spiele“. ${ }^{137}$ Die Sprunghaftigkeit rührt, so Schiller, daher, daß

„sich eine ganz neue Kraft hier in Handlung setzt; denn hier zum erstenmal mischt sich der gesetzgebende Geist in die Handlungen eines blinden Instinktes, unterwirft das willkürliche Verfahren der Einbildungskraft seiner unveränderlichen ewigen Einheit, legt seine Selbständigkeit in das Wandelbare und seine Unendlichkeit in das Sinnliche". ${ }^{138}$

Der Sprung kommt bei Schiller als „ganz neue Kraft“ ins ästhetische Spiel, die der Subsumption des blinden Instinkts und der Willkür der Einbildungskraft unter ein allgemeines Postulat dient: einer auf Einheit abzielenden Konsistenzbildung. Schlegel betont dagegen, daß die Sprunghaftigkeit Symptom je

131 Peirce (Anm. 5), 6.458.

132 Peirce (Anm. 5), 6.458.

133 Peirce (Anm. 5), 6.458.

134 Tatsächlich las Peirce nicht nur über mehrere Jahre hinweg täglich in der Kritik der reinen Vernunft, sondern er beschäftigte sich in jungen Jahren mit Schillers Briefe über die ästhetische Erziehung des Menschen, wie eine Anmerkung aus dem Jahre 1857 belegt. Vgl. Charles Sanders Peirce, Writings of Charles S. Peirce. A Chronological Edition, Volume 1. 1857-1866, M. Fisch, Ch. J. Kloesel, E.C. Moore u.a. (Hrsg.), Bloomington 1982, $10 \mathrm{ff}$.

135 Kant (Anm. 60), 261, fn.

136 Kant (Anm. 60), 262.

137 Friedrich Schiller, Über die Ästhetische Erziehung des Menschen, in: Sämtliche Werke, hrsg. G. Fricke, H.G. Göpfert, München 1967, V, $663 \mathrm{f}$.

138 Schiller (Anm. 137), 663f. ner Bindungsfreiheit sei, die die Voraussetzung für die „logische Chemie“ des Witzes ist. Der Strom unseres Bewußtseins folgt, wie Schlegel in Von der natürlichen Gedankenfolge schreibt, den Assoziationsgesetzen Kontiguität und Similarität. Doch bleibt „der Freiheit und willkürlichen Selbstbestimmung des Menschen ein großer Spielraum offen ", ${ }^{139}$ Die Freiheit der willkürlichen Selbstbestimmung erscheint als chaotische Regellosigkeit der Einbildungskraft, doch auch ihr unterliegt ein Gesetz, nämlich das "genetische Gesetz" des "Überspringens in das Gegenteil". ${ }^{140}$ So besitzt die Einbildungskraft die Macht, „sich mit raschem, kühnen Fluge auf die höchste Höhe der Gedanken zu erheben, und dann plötzlich auf das Entgegengesetzte überzuspringen ". ${ }^{141}$ Dieser „plötzliche Wechsel“ in Form des Sprungs ins Gegenteil beschreibt eine Form der Ambivalenz, die, indem sie einen Gedanken ins Gegenteil verkehrt, entweder eine überraschende Kohärenz oder eine überraschende Inkohärenz herstellt.

Auch für Peirce sind Sprung und Spiel die treibenden Kräfte des Erkenntnisprozesses - und zwar nicht nur des ästhetischen, sondern auch des wissenschaftlichen: Die Wissenschaft kommt hauptsächlich durch Sprünge (leaps) voran, „and the impulse for each leap is either some new observational resource, or some novel way of reasoning about the observations". ${ }^{142}$ Die "neue Kraft" der Erkenntnis ist entweder eine neue Erkenntnisquelle oder ein neuer Denkweg, sie löst entweder innerhalb eines bestehenden Paradigmas oder aber als Paradigmenwechsel Erkenntnissprünge aus. Die epistemologische Pointe des Erkenntnissprungs besteht - ganz im Sinne Kuhns - darin, die Aufmerksamkeit auf bislang unbemerkte Zusammenhänge zu lenken: „[...] it draws attention to relations between facts which would previously have been passed unperceived“. ${ }^{143}$ Dabei wird der „sprunghafte“ Modus der Aufmerksamkeitsverschiebung zumeist von einem „spielerischen“ begleitet, nämlich dem $\mathrm{Ge}$ dankenspiel.

Das Musement beginnt mit einem „vagen Eindruck“, den man an irgendeiner Stelle in einem der drei Universen gewinnt. Gesucht werden sowohl neue Transformationsmöglichkeiten zwischen den verschiedenen Bereichen als auch neue assoziative Zusammenhänge innerhalb eines Bereichs. Die Spezifikation und Intensivierung unserer Beobachtungen und Reflexionen können aus dem Gedankenspiel, etwa dem imaginären „castle building ", ${ }^{144}$ wissenschaftliches

139 Friedrich Schlegel, Von der natürlichen Gedankenfolge, in: Kritische FriedrichSchlegel-Ausgabe, hrsg. Ernst Behler, Hans Eichner, München, Paderborn, Wien 1964, XIII, 290.

140 Schlegel (Anm. 139), 293.

141 Schlegel (Anm. 139), 296.

142 Peirce (Anm. 5), 1.109

143 Peirce (Anm. 5), 1.109

144 Peirce (Anm. 5), 6.458. 
Studium werden lassen. ${ }^{145}$ Sie können aber auch bloßes ästhetisches Spiel bleiben. Insofern ist ein „wildes Spiel der Vorstellungen“ („wild play of the imagination") das „Vorspiel der eigentlichen Wissenschaft“ ${ }^{146}$ Das Gedankenspiel wird damit zur Grundlage einer außerhalb und innerhalb der Wissenschaften wirksamen Phantasie des Neuen, die für Peirce im „Bauen von Luftschlössern“ besteht:

"every man who does accomplish great things is given to building elaborate castles in the air and then painfully copying them on solid ground. Indeed, the whole business of ratiocination, and all that makes us intellectual beings, is performed in imagination ". ${ }^{147}$

Ratiocination ist für Peirce nicht nur ein Synonym für das Produzieren von Inferenzen ${ }^{148}$ sondern bezeichnet auch den Transformationsprozeß, durch den der vormethodische Instinkt in die methodische Rationalität der logischen und ökonomischen Prinzipien erfolgreichen Schlußfolgerns moduliert wird. Dieser Transformationsprozeß muß den Umweg über die Imagination nehmen, um innovativ zu werden. In die gleiche Richtung zielen Ernst Machs Überlegungen zur Psychologie der Forschung, die er in Erkenntnis und Irrtum zeitgleich mit Peirce entfaltet:

Der Projektenmacher, der Erbauer von Luftschlössern, der Romanschreiber, [...] der Dichter sozialer oder technischer Utopien experimentiert in Gedanken. Aber auch der solide Kaufmann, der ernste Erfinder oder Forscher tur dasselbe. Alle stellen sich Umstände vor, und knüpfen an diese Vorstellung die Erwartung, Vermutung gewisser Folgen; sie machen eine Gedankenerfahrung. Während aber die ersteren in der Phantasie Umstände kombinieren, die in Wirklichkeit nicht zusammentreffen, oder diese Umstände von Folgen begleitet denken, welche nicht an dieselben gebunden sind, werden letztere, deren Vorstellungen gute Abbilder der Tatsachen sind, in ihrem Denken der Wirklichkeit sehr nahe bleiben. ${ }^{149}$

Offensichtlich ist die Annahme, daß es eine den Wissenschaften und Künsten gemeinsame Phantasie des Neuen gibt, nicht nur für die Romantiker „um 1800“, sondern auch für die Wissenschaftstheoretiker „um 1900“ eine Selbstverständlichkeit. Eine interessante Anschlußmöglichkeit eröffnen Freuds Überlegungen zu Der Dichter und das Phantasieren. Dort heißt es: Wer phantasiert „,baut sich Luftschlösser, schafft das, was man Tagträume nennt“. ${ }^{150} \mathrm{In}$ teressant ist diese Anschlußmöglichkeit nicht nur wegen der an Peirce und Mach erinnernden Verknüpfung von Phantasie, Luftschloß und Tagtraum,

145 Peirce (Anm. 5), 6.459.

146 Peirce (Anm. S), 1.235.

147 Peirce (Anm. 5), 6.286.

148 Peirce (Anm. 5), 4.39

149 Ernst Mach, Erkenntnis und Irrtum. Skizzen zur Psychologie der Forschung, Leipzig, 3. Aufl.1917, $186 \mathrm{ff}$.

150 Freud (Anm. 1), 172. sondern weil Freud die Logik des Tagtraums durch die Logik des Traums bestimmt.

$$
\text { VIII. }
$$

Für Freud vollzieht die Traumarbeit die Umwandlung des latenten Traumgedankens in einen manifesten. Die Aufgabe der Traumdeutung besteht darin, zu erklären, durch welche Transformationsprozesse (nämlich Verdichtung und Verschiebung) aus dem latenten Traumgedanken der manifeste Traum entsteht. Die Oberflächenstruktur wird als Resultat eines Transformationsprozesses aufgefaßt, der mittels Verdichtung und Verschiebung Wahrnehmungseindrücke aus ihrer bisherigen assoziativen Verkettung herauslöst und aus den freien, „ungebundenen Elementen “ neue assoziative Verknüpfungen herstellt. Diese gehorchen nicht mehr der Logik der Realitätswahrnehmung, sondern der „phantastischen“ Logik des Wunschgedankens. Indem der Traum die „natürlichen “, assoziativen Verknüpfungen auflöst, die als „primary ideas“ der Wahrnehmung und „secondary ideas“ des Denkens unter dem Druck des Realitätsprinzips eingegangen sind, leistet er die Vorarbeit für deren witzige Neuverknüpfung. Der Traum ist sozusagen das Lösungsmittel im Baukasten der witzigen Chemie: Er bewirkt die Verflüssigung starrer Verknüpfungen, die Lockerung fester Kopplungen und gibt so „Freiheit zu neuer Gestaltung“. In Anlehnung an Jean Pauls Witztheorie könnte man sagen: Nur vom Druck des Realitätsprinzips „entbundne Ideen schaffen neue “. ${ }^{151}$ Tatsächlich betont Freud die Analogie zwischen "Traumarbeit" und „Witzarbeit". Insbesondere der Verdichtungsvorgang beim Traum zeigt „mit dem der Witztechnik die größte Ähnlichkeit“. ${ }^{152}$

Während der Traumarbeit erfährt das Material der Traumgedanken „eine ganz außerordentliche Zusammendrängung oder Verdichtung. Ausgangspunkte derselben sind die Gemeinsamkeiten, die sich zufällig oder dem Inhalt gemäß innerhalb der Traumgedanken vorfinden “. ${ }^{153}$ Der zweite Umwandlungsvorgang ist die Traumverschiebung: „Dieselbe äußert sich darin, daß im manifesten Traum zentral steht und mit großer sinnlicher Intensität auftritt, was in den Traumgedanken peripherisch lag und nebensächlich war; und ebenso umgekehrt". ${ }^{154}$ Der Traum leistet also sowohl einen "innovativen Tausch" von Ähnlichkeiten als auch eine „Umwertung der Werte“, was die Relevanzstrukturen betrifft: Das Realitätsprinzip wird marginalisiert, während das Wunschdenken eine zentrale Rolle beansprucht, die ihm freilich immer wieder streitig

151 Jean Paul (Anm.94), 200.

152 Sigmund Freud, Der Witz und seine Beziehung zum Unbeurussten, in: Studienausgabe, Psychologische Schriften, Frankfurt a.M. 1970, IV, 31.

153 Freud (Anm. 152), 153.

154 Freud (Anm. 152), $153 \mathrm{f}$. 
gemacht wird. So deuten die Verschiebungen, etwa die „Ablenkung vom Gedankengang “ bei der Traumarbeit, auf die Einwirkung der Zensur des bewußten, „kritischen“ Denkens hin.

Der Unterschied zwischen Witz und Traum besteht darin, daß der Witz, anders als der Traum, keine Kompromisse eingeht: „er weicht der Hemmung nicht aus, sondern er besteht darauf, das Spiel mit dem Wort oder dem Unsinn unverändert zu erhalten “. ${ }^{155}$ Der Traum ist ein „unkenntlich gemachter Wunsch“, der Witz „ein entwickeltes Spiel“, das nicht nur der Unlustersparnis, sondern explizit dem Lusterwerb dient. ${ }^{156}$ Die Motivation des Witzes liegt in der Suche nach Quellen der Lust, welche aus der Möglichkeit des freien Spiels und des Unsinns hervorgehen. Die gemeinsame Tendenz des Witzes und des Spiels besteht darin, sich „dem Drucke der kritischen Vernunft zu entziehen ", 157 um so eine „Ersparnis an Hemmungsaufwand“ zu erzielen. Dabei bedient sich der Witz genau wie die abduktive Konjektur eines Mittels der Verknüpfung, welches, wie Freud bemerkt, „vom ernsthaften Denken verworfen und sorgfältig vermieden wird ". ${ }^{158}$ Gemeint ist die Auslassung der hinführenden Gedankenwege, also das Auslassen der Mittelglieder bzw. die Enthymematisierung des argumentativen Zusammenhangs. Der Witz führt unter einem logischen Gesichtspunkt zu einem unerlaubten und überraschenden Sprung, zu einem inkohärenten Kurzschluß des Denkens, welcher dadurch Lust erzeugt, daß er gedankliche Arbeit oder „Hemmungsaufwand“ erspart.

„Die Witzlust aus solchem,Kurzschluß'scheint auch um so größer zu sein, je fremder die beiden durch das gleiche Wort in Verbindung gebrachten Vorstellungskreise einander sind, je weiter ab sie voneinander liegen, je größer also die Ersparung an Gedankenweg durch technische Mittel des Witzes ausfällt “. ${ }^{159}$

Eben deshalb erscheinen Witz und kreative Abduktion als überraschender Kurzschluß des Denkens, als Gedankenblitz, der, bestimmte Mittelglieder überspringend, den Gedankenweg abkürzt und dennoch eine unvorhersehbare Einheit stiftet. Zugleich zeigt sich hier, daß Freuds Witztheorie eine interessante Modulation jenes Prinzips vornimmt, das nach Peirce die leitende Überlegung der Abduktion ist: dem Ökonomieprinzip. In seiner pragmatischen Lesart ist das Ökonomieprinzip eine rationale Strategie der „Optimierung des Wissens“. Die epistemologische Gewitztheit besteht darin, sich unnötigen Aufwand zu ersparen, um „kostengünstig“ ans Ziel zu kommen. In seiner psychoanalytischen Lesart wird die „Ersparnistendenz“ dagegen zu einer innovativen Strategie des Unbewußten, die die Konventionen der Logik und der Gesellschaft unterläuft, um sich Aufwand zu ersparen, der normalerweise nötig wäre. Die Phantasie

\footnotetext{
155 Freud (Anm. 152), 161.

156 Freud (Anm.152), 169.

157 Freud (Anm.152), 119.

${ }^{158}$ Freud (Anm. 152), 75.

159 Freud (Anm. 152), 114.
}

des Neuen, die sich im Spiel, im Traum, im Witz offenbart, dient weniger dem Erkenntnisgewinn als dem Lustgewinn.

Auch das Verhältnis von Tagtraum und Abduktion erweist sich bei genauerer Betrachtung als ambivalentes. Wie bereits erwähnt, erlaubt der Pragmatismus ,jeden Flug der Imagination“ - worunter auch Tagträume und Gedankenspiele zu verstehen sind - allerdings nur unter der Voraussetzung, daß diese schließlich in einen Erkenntnisgewinn umgemünzt werden können, ${ }^{160}$ andernfalls werden Tagtraum und Gedankenspiel zur "Phantasterei“. So schreibt Kant in seiner Anthropologie in pragmatischer Hinsicht, wer den „Vergleich mit den Gesetzen der Erfahrung habituell unterläßt (wachend träumt)“, werde zum „Grillenfänger" bzw. zum Phantasten: „Unerwartete Anwandlungen des Phantasten heißen Überfälle der Phantasterei (raptus) “. ${ }^{161}$ Bemerkenswerterweise bedeuten die Ausdrücke "raptus“ und „abduction “ beide das gleiche: Entführung. Die abduktive Phantasie - aber eben auch die abduktive Phantasterei - entführen die Imagination in einen neuen Kontext: sei es als Ideen-Rochade, sei es als Aufpfropfung. Das Resultat dieser Gedankenentführung ist nicht immer nur geniale Sinnstiftung, sondern oft auch „originaler Unsinn “. ${ }^{162}$ Als blitzhafte Konjektur eröffnet die Abduktion zwar „neue Perspektiven “, erweist sich mithin als Phantasie des Neuen, steht aber zugleich auch in der Gefahr, daß sich die neue Perspektive nicht als plausible, innovative Theorie erweist, sondern als bloße Phantasterei. ${ }^{163}$ Dies belegt die folgende Geschichte, die Peirce als Beispiel für „höchst unplausible Theorien “ anführt:

Angenommen, eine Lärche wurde vom Blitz getroffen, und jemand, der ein Liebhaber eben dieser Baumart ist, fragt sich, warum es ausgerechnet die Lärche getroffen hat und nicht einen anderen Baum, und er erhält die folgende Erklärung: Vielleicht gibt es dort oben in den Bergen einen Adlerhorst, und vielleicht hat der männliche Vogel, um sein Nest zu bauen, einen Ast benutzt, in dem ein Nagel steckte. Und einer der kleinen Adler hat sich vielleicht an dem Nagel verletzt, so daß Mutter Adler Vater Adler dafür getadelt hat, daß er einen so gefährlichen Ast benutzte. Er, verärgert von ihren Vorwürfen, mag sich dazu entschlossen haben, den Ast weit weg zu bringen. Und während er unterwegs war, begann das Gewitter. Der Blitz schlug in den Nagel ein und wurde vom Eisen so abgeleitet, daß er die Lärche traf. Natürlich ist dies nur eine Annahme, aber um herauszufinden, warum der Baum getroffen wurde, sollte man sich auf die Suche nach dem Adlerhorst machen. ${ }^{164}$

Das „überraschende Phänomen “ wird hier nicht mit einer instinktgeleiteten, plausiblen Theorie erklärt, sondern mit einem ebenso überraschenden wie in-

\footnotetext{
160 Peirce (Anm. 5), 5.196.

161 Kant (Anm. 13), 513

162 Kant (Anm. 58), 242.

163 Dieses „Kippen“ ins Unplausible kann jedoch auch die Form der „abduktiven Dummheit" annehmen. Vgl. Uwe Wirth, Diskursive Dummheit. Abduktion und Komik als Grenzphänomene des Verstehens, Heidelberg 1999, $155 \mathrm{f}$.

164 Peirce (Anm.5), 2.662, meine Übersetzung.
} 
geniösen Erklärungsversuch verknüpft. Dieser gründet zwar auch auf einer „kreativen Abduktion“, ist aber nicht als wissenschaftliche Hypothese zu werten, da er sich nicht überprüfen läßt. Bei der oben angeführten „narrativen $\mathrm{Hy}$ pothese" handelt es sich um einen Erklärungsversuch, der so innovativ ist, daß er als poetische Phantasterei gewertet werden muß. Entscheidend für unsere Fragestellung ist jedoch ein anderer Umstand, nämlich daß sich die „creation of hypothesis“ im Rahmen der Wissenschaften und im Rahmen der Künste gleichermaßen abduktiven Operationen verdankt, die als "grand work of poietic genius " zu werten sind, ${ }^{165}$ auch wenn sie in beiden Domänen vor dem Hintergrund verschiedener Geltungsansprüche bewertet werden. Mit anderen Worten: Die Abduktion ist eine Phantasie des Neuen, die gleichsam ,an der Schwelle“ zwischen Wissenschaft und Kunst operiert, sozusagen als Übergangsphantasie.

165 Peirce (Anm. 5), 4.238. 